\title{
Prognostic and predictive impacts of tumor-infiltrating lymphocytes differ between Triple-negative and HER2-positive breast cancers treated with standard systemic therapies
}

\author{
Akira I. Hida ${ }^{1,2}$ - Yasuaki Sagara ${ }^{3}$ Daisuke Yotsumoto ${ }^{3}$ - Shuichi Kanemitsu ${ }^{3}$. \\ Junko Kawano $^{3}$ - Shinichi Baba ${ }^{3}$ - Yoshiaki Rai ${ }^{3}$ Y Yumi Oshiro ${ }^{1} \cdot$ Kenjiro Aogi $^{4}$. \\ Yoshiaki Sagara $^{3} \cdot$ Yasuyo Ohi $^{2}$
}

Received: 5 April 2016/Accepted: 28 May 2016/Published online: 3 June 2016

(C) The Author(s) 2016. This article is published with open access at Springerlink.com

\begin{abstract}
Tumor-infiltrating lymphocytes (TILs) have potential value for stratifying the treatment of breast cancer (BC), though their precise use remains unclear. We aimed to investigate the utility of TILs using an alternative approach in different settings. We reviewed patients with triple-negative (TN) or human epithelial growth factor receptor 2 (HER2)-positive invasive ductal carcinomas from a single institutional cohort and classified archived hematoxylineosin-stained samples in terms of TIL score as low $(<10 \%)$, intermediate, and high $(>50 \%)$. The prognostic and predictive values of TILs were analyzed retrospectively in both adjuvant and neo-adjuvant settings. In the adjuvant setting, the presence of TILs at primary surgery was a significant favorable prognostic factor among 154 TNBCs [relapse-free survival (RFS): $p=0.015$ ], but not among 183 HER2+ BCs (RFS: $p=0.097$ ). The TNBC low-TIL group tended to relapse earlier. In the neo-adjuvant setting, detection of TILs on biopsy before primary systemic therapy was associated with the ratio of patients achieving pathological complete
\end{abstract}

Electronic supplementary material The online version of this article (doi:10.1007/s10549-016-3848-2) contains supplementary material, which is available to authorized users.

Akira I. Hida

gbears@matsuyama.jrc.or.jp

1 Department of Pathology, Matsuyama Red Cross Hospital, 1 Bunkyo, Matsuyama, Ehime 790-8524, Japan

2 Department of Pathology, Hakuaikai Sagara Hospital, Kagoshima, Japan

3 Department of Breast Surgical Oncology, Hakuaikai Sagara Hospital, Kagoshima, Japan

4 Department of Breast Oncology, National Hospital Organization Shikoku Cancer Center, Matsuyama, Ehime, Japan response among 48 TNBCs $(p=0.024)$, but not among 58 HER2+ BCs $(p=0.30)$. The presence of TILs in surgical specimens after systemic therapy had prognostic value in HER2+ BC (RFS: $p=0.007)$. The impact of TILs differs between patients with TN and HER2+ BC treated with standard therapies. Our three-grade scale for TILs may contribute to our understanding of the importance of the tumor microenvironment in routine practice. TILs after primary systemic therapy may be useful for the further stratification of treatment of HER2+ BC.

Keywords Tumor-infiltrating lymphocyte - Triplenegative $\cdot$ HER2-positive $\cdot$ Breast cancer

\author{
Abbreviations \\ TIL Tumor-infiltrating lymphocyte \\ BC Breast cancer \\ TN Triple-negative \\ HER2 Human epithelial growth factor receptor 2 \\ RFS Relapse-free survival \\ pCR Pathological complete response \\ ER Estrogen receptor \\ PgR Progesterone receptor \\ HE Hematoxylin-eosin \\ NAC Neoadjuvant chemotherapy \\ IQR Interquartile range \\ LPBC Lymphocytic-predominant breast cancer
}

\section{Background}

The choice of systemic therapy for breast cancer (BC) is based on the pathological diagnosis, which includes histological classification, grade, estrogen receptor (ER) 
status, progesterone receptor $(\mathrm{PgR})$ status, overexpression of human epithelial growth factor receptor 2 (HER2), and Ki67 [1]. Although all these factors provide information on the cancer itself, the microenvironment around a tumor has also been found to be critical for the survival, invasive growth, and metastasis of the cancer.

The host immune system responds to fight cancer. Abnormal antigens on cancer cells can be detected by dendritic cells or macrophages, which activate anti-cancer responses such as cytotoxic T-lymphocytes to eliminate the cancer cells. Tumor-infiltrating lymphocytes (TILs) have shown prognostic value in various kinds of cancers [2], and immune-checkpoint inhibitors have highlighted their potential in treating malignant melanoma and other cancers [3]. This new type of targeted therapy enhances the body's own anti-tumor immunity by releasing the brake caused by the cancer cells [4].

There is increasing evidence regarding the prognostic and predictive values of TILs, mainly in triple-negative (TN) and HER2-positive (HER2+) BCs [5-7]. However, different studies have used different criteria to evaluate TILs; some applied lymphocytic-predominant breast cancer (LPBC: e.g., stromal TILs >50 \%) [6, 8], while others used different thresholds [7, 9]. A standard method is therefore needed to evaluate TILs to allow an integrated discussion and further our understanding of the BC microenvironment. In addition, the association between TILs and trastuzumab-containing chemotherapy in HER2+ $\mathrm{BC}$ is also controversial [10, 11].

An international TIL working group recommended a candidate scoring method [12], but this is too detailed for routine practice. We therefore modified this method and investigated the utility of an alternative simple scoring system for TILs involving hematoxylin-eosin (HE) staining, and explored the clinical values of this scoring in $\mathrm{TN}$ and HER2+ BCs.

\section{Methods}

\section{Patients and samples}

This retrospective, single-institution study was conducted at Sagara Hospital, with ethics committee approval (\#1406). We searched the clinical database for patients with primary BC treated between 2007 and 2014. Patients were included if they had TN-subtype invasive ductal carcinomas (ER - , PgR - , and HER2-) or HER2+ subtype (any $\mathrm{ER}$, any $\mathrm{PgR}$, and HER2+). In the initial study in an adjuvant setting, we included patients who underwent curative surgery and standard postoperative therapy. Patients with small tumors (pT1a) or locally advanced tumors (pT4) were excluded. For the second study in a neo- adjuvant setting, the inclusion criterion was patients with stage I-III cancer treated with standard preoperative chemotherapy. We regarded treatment with an anthracycline or taxane, or an anthracycline followed by a taxane, and with a standard chemotherapy dose as standard systemic therapies for TNBC, and trastuzumab-containing regimens for $\mathrm{HER} 2+\mathrm{BC}$.

The following clinicopathological information was obtained from the database: age; tumor size; histological classification; grade; lymphovascular invasion; nodal status; response to neoadjuvant chemotherapy (NAC); and biomarker profile. The thresholds for ER- and PgR-positivity were set at $1 \%$ using immunohistochemical staining. Stored HE samples were retrieved for TIL assessment. Bilateral tumors, cases with massive necrosis, or tiny residual cancers after NAC were excluded. The REMARK (REporting of tumor MARKer Studies) guidelines were followed [13].

\section{Assessment of TILs using a three-grade scale}

All HE samples were reviewed by a breast pathologist (A.H.) who was blinded to the patient profiles. In resected specimens, a representative slide containing relatively high amounts of both invasive cancer and lymphocytic infiltration was selected for each case. The whole slide was screened using a low-power field, and a "hot spot" with many lymphocytes was identified. TILs were then evaluated in a medium-power field $(100 \times)$ using a three-grade scale (Fig. 1a-c). The region of interest was restricted to within the tumor borders (Fig. 1d-f), as described by Salgado et al. [12]. Lymphocytes and plasma cells were counted, but neutrophils, eosinophils, and macrophages were not. We defined TIL score as the proportion of the area infiltrated by lymphocytes within the tumor itself plus the adjacent stroma, and classified the scores as low $(<10 \%)$, intermediate $(10-50 \%)$, and high (>50\%). Two cut-off points (10 and $50 \%$ ) were chosen from four candidate values $(1,10,30$, and $50 \%)$, because this combination had potential prognostic power and was also fast and easy to implement, as shown in our previous preliminary report [14].

\section{Outcome measures of pathologic complete response and prognosis}

Pathological complete response (pCR) was defined as no residual invasive carcinoma at the primary site, such as ypT0 or ypTis, regardless of lymph node status. Prognostic information was obtained retrospectively from the clinical database and electronic charts. Relapse-free survival (RFS) was defined as the period from surgery to the latest followup, relapse of breast cancer, or death from any cause. 

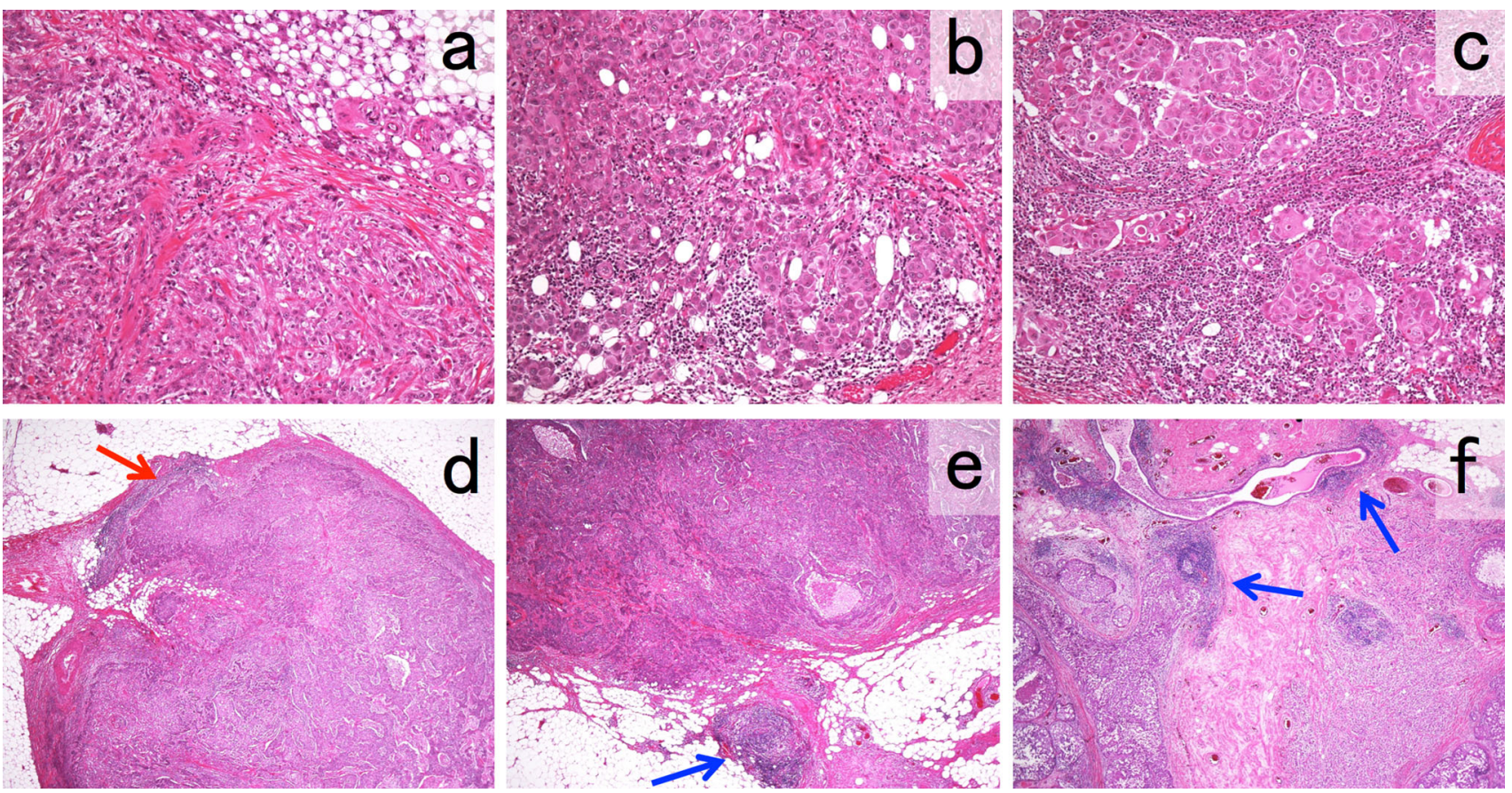

Fig. 1 Representative photomicrographs of HE-stained tissue sections illustrating the simple scoring method for tumor-infiltrating lymphocytes (TILs). The area proportion of TILs was defined as low (a $<10 \%$ ), intermediate (b 10-50\%), or high $(\mathbf{c}>50 \%)$ in a medium-power field $(\times 100 \mathbf{a}-\mathbf{c})$. This score was evaluated in a hot

\section{Statistical analysis}

Survival curves were drawn using the Kaplan-Meier method and analyzed by log-rank tests. The proportions of patients with pCR were compared using $\chi^{2}$ tests. The relationship between TILs and RFS in the adjuvant setting was examined using a Cox proportional hazards model. The limited numbers of recurrent and death events in the neo-adjuvant setting did not allow us to adjust for other prognostic factors using multivariate analysis. Data were analyzed using GraphPad Prism 5 (GraphPad Software, San Diego, CA, USA) and JMP 12 (SAS Institute Inc., Cary, NC, USA).

\section{Results}

\section{Patient characteristics}

A total of 3547 invasive carcinomas were identified from the database, most of which (2598 cases, $73.2 \%$ ) were ER+/HER2-. ER+/HER2+, ER-/HER2+, and TN subtype accounted for $301(8.5 \%), 267(7.5 \%)$, and 381 $(10.7 \%)$ cases, respectively. Based on the criteria described in Patients and samples above, we selected 154 consecutive cases of TNBC and 183 HER2+ BCs in the spot (d, red arrow) infiltrated by many lymphocytes within the border of an invasive tumor. An inflammatory reaction occurring distantly from the tumor (e, blue arrow) or around the intraductal component (f, blue arrow) was not taken into account. A necrotic area was also excluded from a subject

adjuvant setting (Table 1), and 50 consecutive TNBCs and 72 HER2 + BCs in the neo-adjuvant setting (Table 2). These patients were all women aged $22-82$ years. The detailed distributions of age, tumor size, histological grade, ER status, nodal status, regimens of systemic therapy, and pCR ratio are summarized in Tables 1 and 2.

\section{Prognostic value of TILs in the adjuvant setting}

Twenty-five recurrences and 19 deaths were observed after curative surgery and standard postoperative therapy, during a median follow-up period of 45 months [interquartile range (IQR): 24-66.8] for TNBC, and 12 recurrences and five deaths during 58 months (IQR: 44.5-74) for HER2+ BC. TILs (low, intermediate, and high) proved to have significant prognostic value $(p=0.015)$ regarding RFS in TNBC (Fig. 2a), but this was not verified $(p=0.097)$ among HER2+ BCs (Fig. 2b). The prognosis was also significantly poorer in TNBC patients in the low-TIL group compared with the intermediate/high-TIL groups [hazard ratio (HR): 2.68; $95 \%$ confidence interval (CI): 1.13-5.95]. This remained significant in multivariate analysis (HR: 2.49; $95 \%$ CI: 1.05-5.55), as did nodal status (Table 3). Other factors including age, tumor size, histological grade, and lymphovascular invasion had no significant influence on prognosis. 
Table 1 Patient characteristics in the adjuvant setting

\begin{tabular}{|c|c|c|}
\hline & $\mathrm{TN}(n=154)$ & $\operatorname{HER} 2+(n=183)$ \\
\hline \multicolumn{3}{|l|}{ Age } \\
\hline Median (range) & $56.0(22-79)$ & $54.0(31-82)$ \\
\hline$\leq 50$ years & $49(32 \%)$ & $64(35 \%)$ \\
\hline$>50$ years & $105(68 \%)$ & $119(65 \%)$ \\
\hline \multicolumn{3}{|l|}{ Tumor size } \\
\hline pT1b-c & $93(60 \%)$ & $84(46 \%)$ \\
\hline pT2 & $59(38 \%)$ & $91(50 \%)$ \\
\hline pT3 & $2(1 \%)$ & $8(4 \%)$ \\
\hline \multicolumn{3}{|l|}{ Histological grade } \\
\hline HG 1 & $5(3 \%)$ & $4(2 \%)$ \\
\hline HG 2 & $44(29 \%)$ & $105(57 \%)$ \\
\hline HG 3 & $105(68 \%)$ & $74(40 \%)$ \\
\hline \multicolumn{3}{|l|}{ ER status } \\
\hline Negative & $154(100 \%)$ & $92(50 \%)$ \\
\hline Positive & - & $91(50 \%)$ \\
\hline \multicolumn{3}{|l|}{ Lymphovascular invasion } \\
\hline Absent & $100(65 \%)$ & $93(51 \%)$ \\
\hline Present & $54(35 \%)$ & $90(49 \%)$ \\
\hline \multicolumn{3}{|l|}{ Lymph node status } \\
\hline Negative & $98(64 \%)$ & $104(57 \%)$ \\
\hline Positive & $56(36 \%)$ & $79(43 \%)$ \\
\hline \multicolumn{3}{|l|}{ Adjuvant chemotherapy ${ }^{\mathrm{a}}$} \\
\hline Anthracycline & $70(45 \%)$ & $96(52 \%)$ \\
\hline Docetaxel & $24(16 \%)$ & $18(10 \%)$ \\
\hline Paclitaxel & $14(9 \%)$ & $16(9 \%)$ \\
\hline Anthracycline + docetaxel & $18(12 \%)$ & $24(13 \%)$ \\
\hline Anthracycline + paclitaxel & $28(18 \%)$ & $29(16 \%)$ \\
\hline \multicolumn{3}{|l|}{ Endocrine therapy ${ }^{\mathrm{b}}$} \\
\hline Non-steroidal antiestrogens & - & $32(35 \%)$ \\
\hline Aromatase inhibitors & - & $28(31 \%)$ \\
\hline Both of above & - & $20(22 \%)$ \\
\hline
\end{tabular}

$T N$ triple-negative, $H E R 2$ human epithelial growth factor receptor 2, $p T 1-3$ according to the 7th edition of the UICC, ER estrogen receptor

a Trastuzumab was also administered to all HER2+ cases

b Eleven HER2+/ER + cases operated on during 2007-2010 did not receive endocrine therapy because of low ER expression (1-10\%)

\section{Association between TILs in biopsy samples before NAC and pCR ratio}

Biopsy samples taken before NAC were available for 48 TNBCs and 58 HER2+ BCs. Four cases only had intraductal carcinomas on core needle biopsies, and 12 other biopsies were unavailable because they had been diagnosed at other institutions. TILs were evaluated with the same method using the three-grade scale, and the score was compared with the pCR ratio. TILs were significantly related to pCR ratio in TNBC $(p=0.024)$ (Fig. 3a), but not in HER2+ BC $(p=0.30)$ (Fig. 3b).

\section{Prognostic value of TILs in resected specimens after NAC}

Among resected specimens after NAC, 28 TNBC and 41 HER2+ BC non-pCR cases underwent TIL assessment. One TNBC case was excluded because the residual cancer was only detected in the lymphatic vessels. For TNBC, 11 recurrences and seven deaths were observed during a 23-month median follow-up period (IQR: 12.5-37), and 10 recurrences and four deaths were observed during 34 months (IQR: 23.8-48.3) for HER2+ BC. The low- and intermediate-TIL groups showed relatively poorer prognoses than the high-TIL group in TNBC (Fig. 4a), though the differences were not statistically significant $(p=0.24)$. The three-grade scale for TILs revealed significant prognostic differences in HER2+ BC (RFS: $p=0.007$ ) (Fig. 4b).

In addition, 26 and 32 pair-matched TN and HER2+ BC samples were available, respectively. The TIL scores of pre-treatment biopsy and post-treatment surgical samples were compared (Online Resource). Changes in TIL scores were classified as decrease, increase, and no change. The proportions of recurrent cases in each subgroup are summarized in Fig. 5. The distributions of non-pCR cases were similar in each subgroup. Patients with TNBC and decreased TIL tended to have a higher rate of relapse, but the difference was not significant in this small setting.

\section{Discussion}

The results of this retrospective study verified the prognostic and predictive values of TILs in TNBC, in accordance with other studies [5, 6, 9]. Consistent with a recent report, we found that TILs had no prognostic value concerning HER2 + BC in the adjuvant setting with trastuzumab-containing chemotherapy [11]. The N9831 trial also demonstrated that LPBC was a significant favorable prognostic factor in the adjuvant setting regarding HER2+ $\mathrm{BC}$ treated with chemotherapy alone. The microenvironment of non-LPBC tumors appears to be dramatically improved by adding trastuzumab, such that the prognostic impact of TILs disappears in the trastuzumab-containing setting. This improvement may be explained by trastuzumab's strong antibody-dependent cellular cytotoxicity [15], or by adaptive immune reaction following cancer-cell death induced by trastuzumab-containing chemotherapy [16]. These findings fit well with our results; HER2 + cases with low numbers of TILs might have high levels of lymphocytes in reserve, and trastuzumab-containing systemic therapy could activate this potential. In contrast, some studies have reported that HER2 + BC has a different immune profile [17]. 
Table 2 Patient characteristics in the neo-adjuvant setting

$$
\mathrm{TN}(n=50)
$$

HER2 $+(n=72)$

\section{Age}

Median (range)

$51.5(28-75)$

$58.0(31-81)$

$\leq 50$ years

$>50$ years

$23(46 \%)$

$21(29 \%)$

$27(54 \%)$

$51(71 \%)$

ER status

Negative

$50(100 \%)$

$36(50 \%)$

Positive

Unknown

Tumor size after systemic therapy

ypT1

урт2

урT3

ypT4

pCR (ypT0 \& ypTis)

$-$

$-$

$33(46 \%)$

$-\quad 3(4 \%)$

$16(32 \%)$

$29(40 \%)$

$7(14 \%)$

$9(13 \%)$

$2(4 \%)$

$1(1 \%)$

$4(8 \%)$

$2(3 \%)$

Lymph node status after systemic therapy ${ }^{a}$

Negative

$21(42 \%)$

$31(43 \%)$

Positive

$38(76 \%) \quad 48(67 \%)$

Preoperative therapy

Anthracycline $\rightarrow$ taxane

$12(24 \%)$

$23(32 \%)$

Taxane $\rightarrow$ anthracycline

Anthracycline $\rightarrow$ taxane + trastuzumab

Taxane + trastuzumab

$43(86 \%)$

$7(14 \%)$

$-$

$-$

$-$

Taxane + trastuzumab $\rightarrow$ anthracycline + trastuzumab

Endocrine therapy ${ }^{\mathrm{b}}$

Non-steroidal antiestrogens

Aromatase inhibitors

Both of above

$T N$ triple-negative, $H E R 2$ human epithelial growth factor receptor 2, ypT0-4 according to the 7th edition of the UICC, ER estrogen receptor

a One HER2+ case did not undergo axillary sampling

${ }^{\mathrm{b}}$ Four HER2+/ER + cases with low ER expression (1-10\%) did not receive endocrine therapy

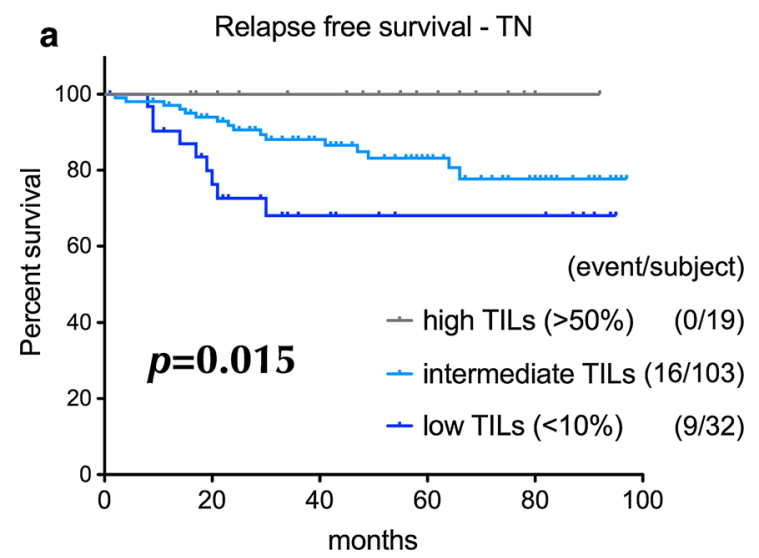

Fig. 2 Prognostic value of tumor-infiltrating lymphocytes (TILs) in the adjuvant setting. Relapse-free survival was analyzed in the adjuvant setting for 154 TNBCs (a) and 183 HER2+ BCs (b). Stored samples obtained from primary surgery were categorized into three

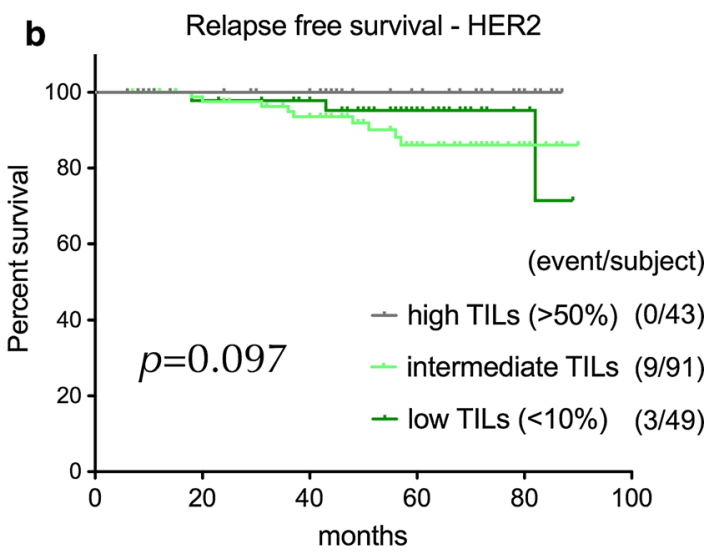

groups of low, intermediate, and high TILs. All patients received standard chemotherapy after curative surgery. $T N$ triple-negative, $B C$ breast cancer, HER2 human epithelial growth factor receptor 2 
Table 3 Association of tumorinfiltrating lymphocytes with prognosis of relapse-free survival in patients with triplenegative breast cancer

\begin{tabular}{|c|c|c|c|}
\hline & \multirow{2}{*}{$\begin{array}{l}\text { Univariate analysis } \\
\text { HR }(95 \% \mathrm{CI})\end{array}$} & \multicolumn{2}{|c|}{ Multivariate analysis } \\
\hline & & $\mathrm{HR}(95 \% \mathrm{CI})$ & $p$ \\
\hline \multicolumn{4}{|l|}{ TILs } \\
\hline Low versus intermediate/high & $2.68(1.13-5.95)$ & $2.49(1.05-5.55)$ & 0.039 \\
\hline \multicolumn{4}{|l|}{ Age } \\
\hline$\leq 50$ years versus $>50$ years & $1.21(0.51-2.69)$ & - & - \\
\hline \multicolumn{4}{|l|}{ Tumor size } \\
\hline$>2 \mathrm{~cm}$ versus $\leq 2 \mathrm{~cm}$ & $1.37(0.61-3.02)$ & - & - \\
\hline \multicolumn{4}{|l|}{ Histological grade } \\
\hline 3 versus $1 / 2$ & $1.78(0.72-5.34)$ & - & - \\
\hline \multicolumn{4}{|l|}{ Lymphovascular invasion } \\
\hline Present versus absent & $1.83(0.82-4.07)$ & - & - \\
\hline \multicolumn{4}{|l|}{ Nodal status } \\
\hline Positive versus negative & $2.85(1.28-6.76)$ & $2.71(1.22-6.42)$ & 0.015 \\
\hline
\end{tabular}

TILs tumor-infiltrating lymphocytes, TNBC triple-negative breast cancer $H R$ hazard ratio; $C I$ confidence interval
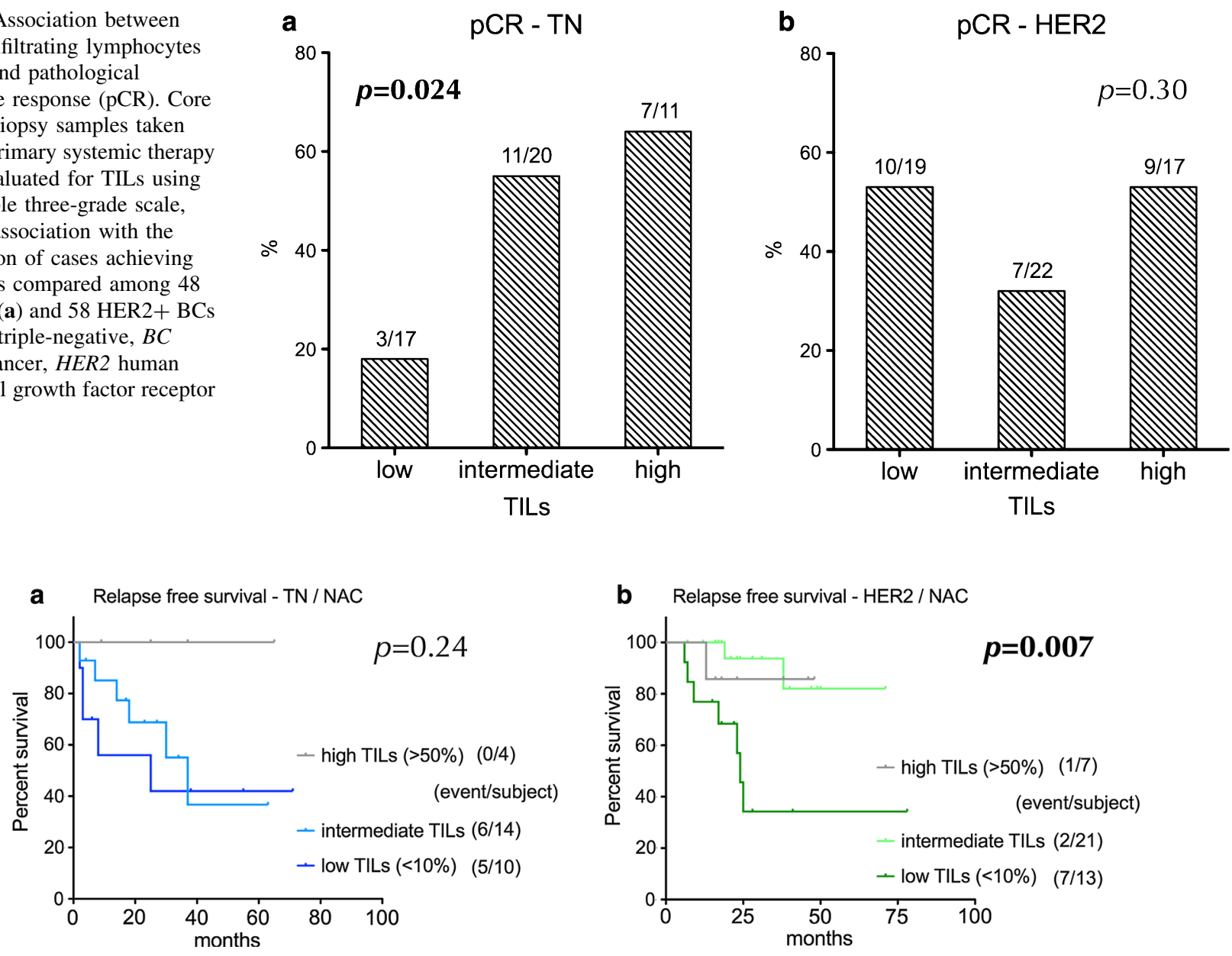

Fig. 4 Survival analysis after neo-adjuvant systemic therapy. Relapse-free survival was analyzed among 28 TNBCs (a) and 41 HER 2+ BCs (b). About $42 \%$ of each subtype achieved pathological complete response (pCR) after standard neo-adjuvant chemotherapy
(NAC). For non-pCR cases, tumor-infiltrating lymphocytes (TILs) in the residual carcinoma were evaluated in the three groups of low, intermediate, and high TILs. $T N$ triple-negative, $B C$ breast cancer, HER2 human epithelial growth factor receptor 2 
Fig. 5 Influence of change in tumor-infiltrating lymphocyte (TIL) score before and after neo-adjuvant chemotherapy (NAC) on the proportion of recurrent cases. We applied the three-grade scale for TILs to both pre-treatment biopsy samples and post-treatment surgical specimens. Matched pairs of $26 \mathrm{TNBCs}(\mathbf{a})$ and 32 HER2 + BCs (b) were analyzed. $T N$ triple-negative, $B C$ breast cancer, HER2 human epithelial growth factor receptor 2

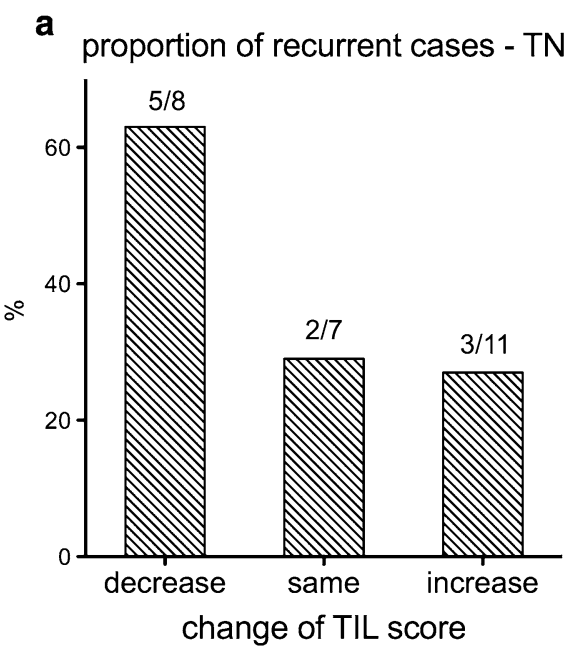

$\begin{array}{lllllll}21.5 & 23.0 & 25.0 & \text { (months) } & 18.0 & 24.0 & 23.5\end{array}$

b
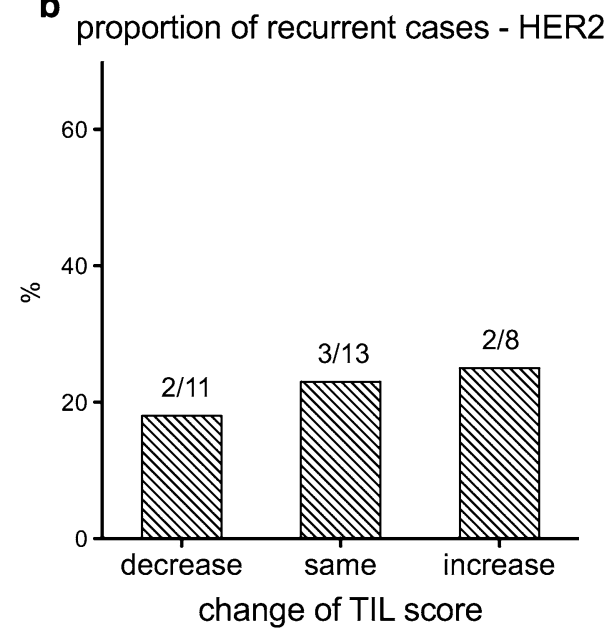

change of TIL score

median follow up time

TILs in residual carcinoma after NAC had a significant prognostic impact among HER2+ BCs. These TILs were evaluated after trastuzumab-containing chemotherapy and had therefore already been influenced by systemic treatment. To the best of our knowledge, this is the first report indicating that TIL status after NAC is a favorable prognostic factor in HER2+ BC. Similarly, Dieci et al. [18] reported the prognostic value of TILs in residual disease after NAC in TNBC. Their survival curves for the HighTIL (stromal and/or intratumoral TIL $>60 \%$ ) and LowTIL groups are similar to those for patients with TNBC in the present study. However, the difference between the high-TIL and low/intermediate-TIL groups was not significant (HR: 3.49; $95 \%$ CI: $0.75-16.3$ ). The lack of statistical power $(n=28)$ and absence of events in the highTIL group may account for the discordant result. The prognostic value of TILs in residual disease therefore needs to be confirmed in a larger cohort.

Our study had several limitations. In terms of analytical validation, we did not prove the robustness and reproducibility of the three-grade scale for TILs. However, intraobserver concordance was good (weighted $\kappa=0.62$ ) in our recent investigation $(n=90$, estimated by A.H., 3-month intervals; detailed data not shown), and inter-observer concordance was also good (weighted $\kappa=0.78$ ) as shown at supplementary Table $\mathrm{S} 1$ in our previous report [14]. However, these data are inadequate, and inter-laboratory concordances should be examined before standardization. Given the limitations on reproducibility associated with human analytical procedures, automated technology using immunohistochemical staining [19] or multi-gene assays [20] may represent possible future directions. It is also unclear if we should evaluate average areas or hot spots. We recognized the heterogeneity of TILs (different intensities of lymphocytes in different areas), and hot spots tended to be located at the invasive edge. Further evidence is therefore needed regarding the role, mechanisms involved, and importance of hot spots. Another limitation was the small number of patients evaluated and the relatively short follow-up period. Nevertheless, we applied a single uniform method throughout the study, which clarified differences in the prognostic value of TILs between $\mathrm{TN}$ and HER2+ BCs, and also between adjuvant and neoadjuvant settings.

New strategies should be considered to improve the outcome of patients with TNBC, such as immune-checkpoint inhibitors in low-TIL patients. TILs have the potential to act as an additional surrogate marker for survival, and TILs in residual carcinoma after NAC may therefore help in assessing risk and stratifying treatment in patients with either TN or HER2+ BC. This rapid and practical method may contribute to further translational research and the discovery of innovative drugs.

\section{Conclusions}

In summary, we propose a simple method to evaluate TILs. Based on a three-grade scale, the clinical value of TILs differs between TN and HER2+ BCs in patients receiving standard therapies. TILs after NAC seem to have a favorable prognostic impact on HER2 $+\mathrm{BC}$ and possibly on TNBC, suggesting that they may represent a surrogate marker for survival and drug efficacy in these situations.

Acknowledgments The authors would like to thank S. Haraguchi at the research center of Sagara Hospital for extracting the necessary information from the database, and the technicians at the Department 
of Pathology at Sagara Hospital for their assistance in dealing with archived samples.

Funding This study was funded by the Division of Clinical Research Promotion at the National Hospital Organization Shikoku Cancer Center.

\section{Compliance with ethical standards}

Conflict of interest Kenjiro Aogi received personal fees as honoraria from Chugai Pharmaceutical, Eisai, Sanofi, SRL, AstraZeneca, Taiho Pharmaceutical, Novartis Pharma, Daiichi Sankyo, Mochida Pharmaceutical, Ono Pharmaceutical, Otsuka Pharmaceutical, Nihon Medi-Physics and Eli Lilly Japan, and his institution received research funds from Chugai Pharmaceutical, Eisai, and Sanofi. All remaining authors have no conflicts of interest to declare.

Ethical approval All procedures performed in this study involving human participants were performed in accordance with the ethical standards of the institutional research committee (No. 14-06) and with the 1964 Helsinki declaration and its later amendments. Formal consent was not required for this type of study.

Open Access This article is distributed under the terms of the Creative Commons Attribution-NonCommercial 4.0 International License (http://creativecommons.org/licenses/by-nc/4.0/), which permits any noncommercial use, distribution, and reproduction in any medium, provided you give appropriate credit to the original author(s) and the source, provide a link to the Creative Commons license, and indicate if changes were made.

\section{References}

1. Coates AS, Winer EP, Goldhirsch A, Gelber RD, Gnant M, Piccart-Gebhart M, Thurlimann B, Senn HJ, Panel M (2015) Tailoring therapies-improving the management of early breast cancer: St Gallen International Expert Consensus on the Primary Therapy of Early Breast Cancer 2015. Ann Oncol 26(8):1533-1546. doi:10.1093/annonc/mdv221

2. Gooden MJ, de Bock GH, Leffers N, Daemen T, Nijman HW (2011) The prognostic influence of tumour-infiltrating lymphocytes in cancer: a systematic review with meta-analysis. $\mathrm{Br} \mathrm{J}$ Cancer 105(1):93-103. doi:10.1038/bjc.2011.189

3. Postow MA, Callahan MK, Wolchok JD (2015) Immune checkpoint blockade in cancer therapy. J Clin Oncol 33(17):1974-1982. doi:10.1200/JCO.2014.59.4358

4. Okazaki T, Chikuma S, Iwai Y, Fagarasan S, Honjo T (2013) A rheostat for immune responses: the unique properties of PD- 1 and their advantages for clinical application. Nat Immunol 14(12):1212-1218. doi:10.1038/ni.2762

5. Adams S, Gray RJ, Demaria S, Goldstein L, Perez EA, Shulman LN, Martino S, Wang M, Jones VE, Saphner TJ, Wolff AC, Wood WC, Davidson NE, Sledge GW, Sparano JA, Badve SS (2014) Prognostic value of tumor-infiltrating lymphocytes in triple-negative breast cancers from two phase III randomized adjuvant breast cancer trials: ECOG 2197 and ECOG 1199. J Clin Oncol 32(27):2959-2966. doi:10.1200/JCO.2013.55.0491

6. Dieci MV, Mathieu MC, Guarneri V, Conte P, Delaloge S, Andre F, Goubar A (2015) Prognostic and predictive value of tumorinfiltrating lymphocytes in two phase III randomized adjuvant breast cancer trials. Ann Oncol 26(8):1698-1704. doi:10.1093/ annonc/mdv239
7. Salgado R, Denkert C, Campbell C, Savas P, Nucifero P, Aura C, de Azambuja E, Eidtmann H, Ellis CE, Baselga J, Piccart-Gebhart MJ, Michiels S, Bradbury I, Sotiriou C, Loi S (2015) Tumorinfiltrating lymphocytes and associations with pathological complete response and event-free survival in HER2-positive Early-stage breast cancer treated with lapatinib and trastuzumab: a secondary analysis of the NeoALTTO trial. JAMA Oncol 1(4):448-454. doi:10.1001/jamaoncol.2015.0830

8. Loi S, Sirtaine N, Piette F, Salgado R, Viale G, Van Eenoo F, Rouas G, Francis P, Crown JP, Hitre E, de Azambuja E, Quinaux E, Di Leo A, Michiels S, Piccart MJ, Sotiriou C (2013) Prognostic and predictive value of tumor-infiltrating lymphocytes in a phase III randomized adjuvant breast cancer trial in node-positive breast cancer comparing the addition of docetaxel to doxorubicin with doxorubicin-based chemotherapy: BIG 02-98. J Clin Oncol 31(7):860-867. doi:10.1200/JCO.2011.41.0902

9. Denkert C, Loibl S, Noske A, Roller M, Muller BM, Komor M, Budczies J, Darb-Esfahani S, Kronenwett R, Hanusch C, von Torne C, Weichert W, Engels K, Solbach C, Schrader I, Dietel M, von Minckwitz G (2010) Tumor-associated lymphocytes as an independent predictor of response to neoadjuvant chemotherapy in breast cancer. J Clin Oncol 28(1):105-113. doi:10.1200/JCO. 2009.23.7370

10. Loi S, Michiels S, Salgado R, Sirtaine N, Jose V, Fumagalli D, Kellokumpu-Lehtinen PL, Bono P, Kataja V, Desmedt C, Piccart MJ, Loibl S, Denkert C, Smyth MJ, Joensuu H, Sotiriou C (2014) Tumor infiltrating lymphocytes are prognostic in triple negative breast cancer and predictive for trastuzumab benefit in early breast cancer: results from the FinHER trial. Ann Oncol 25(8):1544-1550. doi:10.1093/annonc/mdu112

11. Perez EA, Ballman KV, Tenner KS, Thompson EA, Badve SS, Bailey H, Baehner FL (2016) Association of stromal tumor-infiltrating lymphocytes with recurrence-free survival in the N9831 adjuvant trial in patients with early-stage HER2-positive breast cancer. JAMA Oncol 2(1):56-64. doi:10.1001/jamaoncol.2015. 3239

12. Salgado R, Denkert C, Demaria S, Sirtaine N, Klauschen F, Pruneri G, Wienert S, Van den Eynden G, Baehner FL, PenaultLlorca F, Perez EA, Thompson EA, Symmans WF, Richardson AL, Brock J, Criscitiello C, Bailey H, Ignatiadis M, Floris G, Sparano J, Kos Z, Nielsen T, Rimm DL, Allison KH, Reis-Filho JS, Loibl S, Sotiriou C, Viale G, Badve S, Adams S, WillardGallo K, Loi S (2015) The evaluation of tumor-infiltrating lymphocytes (TILs) in breast cancer: recommendations by an International TILs Working Group 2014. Ann Oncol 26(2):259-271. doi:10.1093/annonc/mdu450

13. McShane LM, Altman DG, Sauerbrei W, Taube SE, Gion M, Clark GM, Statistics Subcommittee of the NCIEWGoCD (2005) Reporting recommendations for tumor marker prognostic studies. J Clin Oncol 23(36):9067-9072. doi:10.1200/JCO.2004.01.0454

14. Hida AI, Ohi Y (2015) Evaluation of tumor-infiltrating lymphocytes in breast cancer; proposal of a simpler method. Ann Oncol 26(11):2351. doi:10.1093/annonc/mdv363

15. Park S, Jiang Z, Mortenson ED, Deng L, Radkevich-Brown O, Yang X, Sattar H, Wang Y, Brown NK, Greene M, Liu Y, Tang J, Wang S, Fu YX (2010) The therapeutic effect of anti-HER2/neu antibody depends on both innate and adaptive immunity. Cancer Cell 18(2):160-170. doi:10.1016/j.ccr.2010.06.014

16. Bianchini G, Gianni L (2014) The immune system and response to HER2-targeted treatment in breast cancer. Lancet Oncol 15(2):e58-e68. doi:10.1016/s1470-2045(13)70477-7

17. Muraro E, Martorelli D, Turchet E, Miolo G, Scalone S, Comaro E, Talamini R, Mastorci K, Lombardi D, Perin T, Carbone A, Veronesi A, Crivellari D, Dolcetti R (2011) A different immunologic profile characterizes patients with HER-2-overexpressing and HER-2-negative locally advanced breast cancer: 
implications for immune-based therapies. Breast Cancer Res 13(6):R117. doi:10.1186/bcr3060

18. Dieci MV, Criscitiello C, Goubar A, Viale G, Conte P, Guarneri V, Ficarra G, Mathieu MC, Delaloge S, Curigliano G, Andre F (2014) Prognostic value of tumor-infiltrating lymphocytes on residual disease after primary chemotherapy for triple-negative breast cancer: a retrospective multicenter study. Ann Oncol 25(3):611-618. doi:10.1093/annonc/mdt556

19. Miyashita M, Sasano H, Tamaki K, Hirakawa H, Takahashi Y, Nakagawa S, Watanabe G, Tada H, Suzuki A, Ohuchi N, Ishida T (2015) Prognostic significance of tumor-infiltrating CD8+ and FOXP3 + lymphocytes in residual tumors and alterations in these parameters after neoadjuvant chemotherapy in triple-negative breast cancer: a retrospective multicenter study. Breast Cancer Res 17(1):124. doi:10.1186/s13058-015-0632-x

20. Denkert C, von Minckwitz G, Brase JC, Sinn BV, Gade S, Kronenwett R, Pfitzner BM, Salat C, Loi S, Schmitt WD, Schem C, Fisch K, Darb-Esfahani S, Mehta K, Sotiriou C, Wienert S, Klare P, Andre F, Klauschen F, Blohmer JU, Krappmann K, Schmidt M, Tesch H, Kummel S, Sinn P, Jackisch C, Dietel M, Reimer T, Untch M, Loibl S (2015) Tumor-infiltrating lymphocytes and response to neoadjuvant chemotherapy with or without carboplatin in human epidermal growth factor receptor 2-positive and triple-negative primary breast cancers. J Clin Oncol 33(9):983-991. doi:10.1200/JCO.2014.58.1967 